\title{
Circuito CMOS para el control de temperatura de sensores de gas MOX CMOS temperature control circuit for MOX gas sensors
}

\author{
Álvarez-Simón Luis Carlos \\ Universidad Autónoma del Estado de México \\ Correo: alvarez.simon.dr@gmail.com \\ https://orcid.org/0000-0002-1389-3931
}

\author{
Gómez-Ramírez Emmanuel \\ Conacyt-Tecnológico Nacional de México \\ Instituto Tecnológico de La Laguna \\ Correo: egomez.ram@gmail.com \\ https://orcid.org/0000-0001-7104-9520
}

\section{Resumen}

En los últimos años, la contaminación del aire y la detección de gases nocivos se han convertido en una vertiente en la investigación. Por ello, hoy en día se han desarrollado diversos sensores capaces de detectar diferentes gases, por ejemplo, los sensores químicoresistivos y los de tipo metal-óxido-semiconductor (sensores MOX), los cuales se destacan entre otras tecnologías. Los sensores de gas MOX permiten detectar múltiples gases con una alta sensibilidad y además son compatibles con tecnologías de integración CMOS. Los sensores MOX combinan un elemento de sensado de gas y un elemento de calentamiento para la selectividad de gases. Actualmente, la modulación de la temperatura de operación es una de las técnicas más usadas para mejorar la selectividad y estabilidad de los sensores de gas MOX. En este trabajo se propone un circuito de control on/off para la modulación de la temperatura de operación de sensores MOX utilizando la resistencia del calentador para monitorear su temperatura. Este circuito permite aplicar diferentes técnicas de modulación de temperatura tales como la técnica de modulación por pulsos, la técnica de modulación por ondas periódicas y el control a diferentes niveles de la temperatura de operación para la generación de matrices virtuales de sensores mediante un solo sensor. El circuito se diseñó en una tecnología CMOS de $180 \mathrm{~nm}$ y se simuló usando un modelo simple del sensor comercial AS-MLC de AppliedSensor. El circuito propuesto permite alcanzar exactitudes de $0.2 \Omega$ en el valor de la resistencia del calentador, el cual corresponde aproximadamente a un error de $1{ }^{\circ} \mathrm{C}$ en la temperatura de operación del sensor.

Descriptores: Control de temperatura, sensores de gas MOX, modulación de temperatura, circuitos CMOS, Circuito de control on-off.

\begin{abstract}
In the last years, air pollution and the detection of harmful gases has become one important branch in research. For this reason, several sensors capable of detecting different gases have been developed, for example, chemical-resistive sensors and metal-oxide-semiconductor sensors (MOX sensors). MOX gas sensors allow the detection of multiple gases with high sensitivity and are also compatible with CMOS integration technologies. The MOX sensors combine a gas sensing element and a heating element for the gas selectivity. Currently, the modulation of the operating temperature is one of the most used techniques to improve the selectivity and stability of the MOX gas sensors. In this work, an on/off control circuit for temperature modulation in MOX sensors using resistance from Heater to monitor the temperature is proposed. This circuit allows applying different temperature modulation techniques like pulses modulation technique, periodic wave modulation technique, and control at different levels of the operating temperature for the generation of virtual arrays of sensors by a single sensor. The circuit was designed in a 180nm CMOS technology and simulated using a simple model of a commercial sensor AS-MLC from AppliedSensor. The proposed control circuit allows achieving accuracies of $0.2 \Omega$ in the Heater resistance value, which corresponds to an error of $1{ }^{\circ} \mathrm{C}$ in the operating temperature of the sensor approximately. Keywords: Temperature control, MOX gas sensor, temperature modulation, CMOS circuit, on/off control circuit.
\end{abstract}




\section{INTRODUCCIÓN}

Actualmente tanto la contaminación del aire como la detección de gases tóxicos en las industrias y en lugares públicos, han sido un tema de interés para la comunidad científica y social, incrementando la necesidad de monitorear y regular la emisión de ciertos gases que afectan la salud de la población en general (Dongmin et al., 2010; Jendritzky, 1993). Por ello, la implementación de sistemas de monitoreo ha incrementado en los últimos años. Sin embargo, la detección de gases nocivos se sigue realizando con instrumentos de laboratorio, lo que encarece y dificulta este tipo de detección. Una alternativa para subsanar esto es el desarrollo de sistemas de detección de gas portables de bajo costo. Para ello, hoy en día se han desarrollado diversos sensores capaces de detectar diferentes gases, entre los que destacan los sensores químico-resistivos y los de tipo metal-óxido-semiconductor (MOX) (Gardner et al., 2010; Burgues y Marco, 2018; Bharat et al., 2018). Estos últimos combinan, principalmente, un elemento de sensado de gas y un elemento de calentamiento, ya que la sensibilidad y selectividad de la película de sensado es dependiente de su temperatura de operación. Los sensores MOX operan a temperaturas superiores a los $300^{\circ} \mathrm{C}$ (Seiyama et al., 1962). En contraste con los sensores de estado sólido en otras tecnologías, los sensores MOX tienen la capacidad de detectar múltiples gases con una alta sensibilidad, pudiendo sensar niveles tan bajos como partes por billón (ppb), además de una mayor compatibilidad con la tecnología CMOS y por ende un bajo costo, lo que ha motivado a la co-integración con los circuitos de acondicionamiento de señal en un solo circuito integrado (Korotcenkoy, 2007).

Los sensores de gas de tipo MOX aún presentan inconvenientes respecto a su selectividad (Ponzoni, 2017). Además de la constante búsqueda de materiales más selectivos, se ha hecho uso de las características estáticas y dinámicas del propio sensor para mejorar su desempeño, utilizando en algunos casos, matrices de sensores no-selectivos junto con técnicas de reconocimiento de patrones para aumentar la selectividad (Bermak et al., 2005). Otra técnica consiste en discriminar los gases a través de la respuesta dinámica del sensor al variar su temperatura de operación, lo que se conoce como técnica de modulación de temperatura (Lee y Reedy, 1999). Cabe mencionar que este se ha convertido en el método más usado para mitigar la baja selectividad en el desarrollo de sistemas de sensado de gases de bajo costo en los últimos años (Ponzoni, 2017; Bermak et al., 2005).

Para el control de la temperatura de la película sensible, se pueden utilizar dos elementos, uno para sensar la temperatura de la película y otro para calentar (Kang et al., 2017). Sin embargo, es posible usar un solo dispositivo para llevar a cabo ambos procesos, es decir, usando las variaciones de resistencia del propio calentador como sensor de temperatura (Falconi, 2013), lo que conlleva a una reducción del costo de fabricación, siendo este método el que se usa en este trabajo. En la literatura se pueden encontrar diferentes propuestas de circuitos de control de temperatura para sensores de gas MOX, desde esquemas de control simples como el control on/off, hasta algunos más complejos con bloques Proporcional-Integral-Derivativo (PID) (Falconi y Fratini, 2008; Bota et al., 2004; Casanova et al., 2004).

En la Figura 1 se representan de manera general los bloques necesarios para implementar un sistema de sensado de gas basado en sensores de gas MOX con modulación de la temperatura de operación. El sistema tiene dos subsistemas principales: el encargado del control de la temperatura de operación de la película sensible y el que determina la concentración del gas o gases presentes. Este último comprendido por un sensor o conjunto de sensores, un circuito de acondicionamiento $\mathrm{y}$ un bloque de reconocimiento de patrones para determinar la concentración de gases. El bloque del circuito de control de temperatura por su parte debe ser capaz de manipular la temperatura de operación de la película sensible, de tal manera que se puedan aplicar las técnicas de modulación de temperatura que se han propuesto en la literatura para este tipo de sensores, con el fin de mejorar su selectividad (Lee y Reedy, 1999).

En este trabajo se propone un circuito de control de tipo on/off, el cual permite utilizar diferentes técnicas de modulación de temperatura y además permite el uso de sensores de gas MOX con diferentes requerimientos en cuanto a la potencia necesaria para alcanzar la temperatura de operación deseada.

El contenido de este trabajo se distribuye de la siguiente manera: en las siguientes secciones se describe el diseño del circuito de control de temperatura propuesto, se muestran los resultados obtenidos y por último las conclusiones del trabajo.

\section{Ciruito de COntrol propuesto}

El circuito de control de temperatura propuesto se muestra en la Figura 2, el cual utiliza dos voltajes de alimentación, denominados $V_{D D L}$ y $V_{D D H}$ lo que le da la flexibilidad de controlar Heaters con diferentes requerimientos de potencia (Casanova et al., 2004). corresponde al voltaje de alimentación general para los circuitos de acondicionamiento analógico y digital, mientras que 


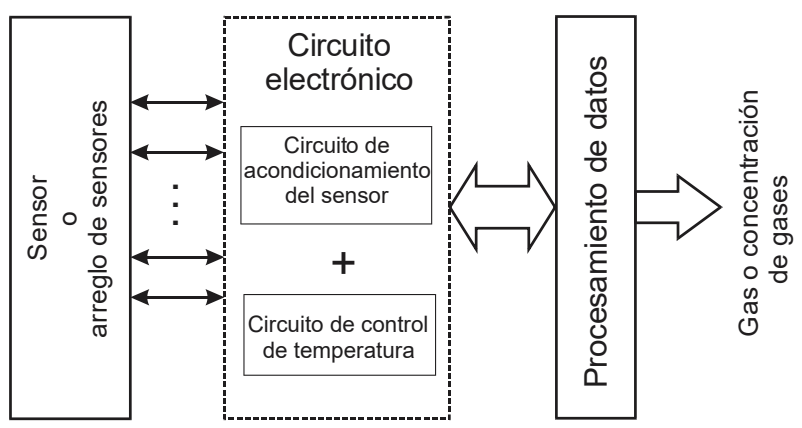

Figura 1. Esquema simplificado de un sistema de sensado de gas basado en sensores MOX

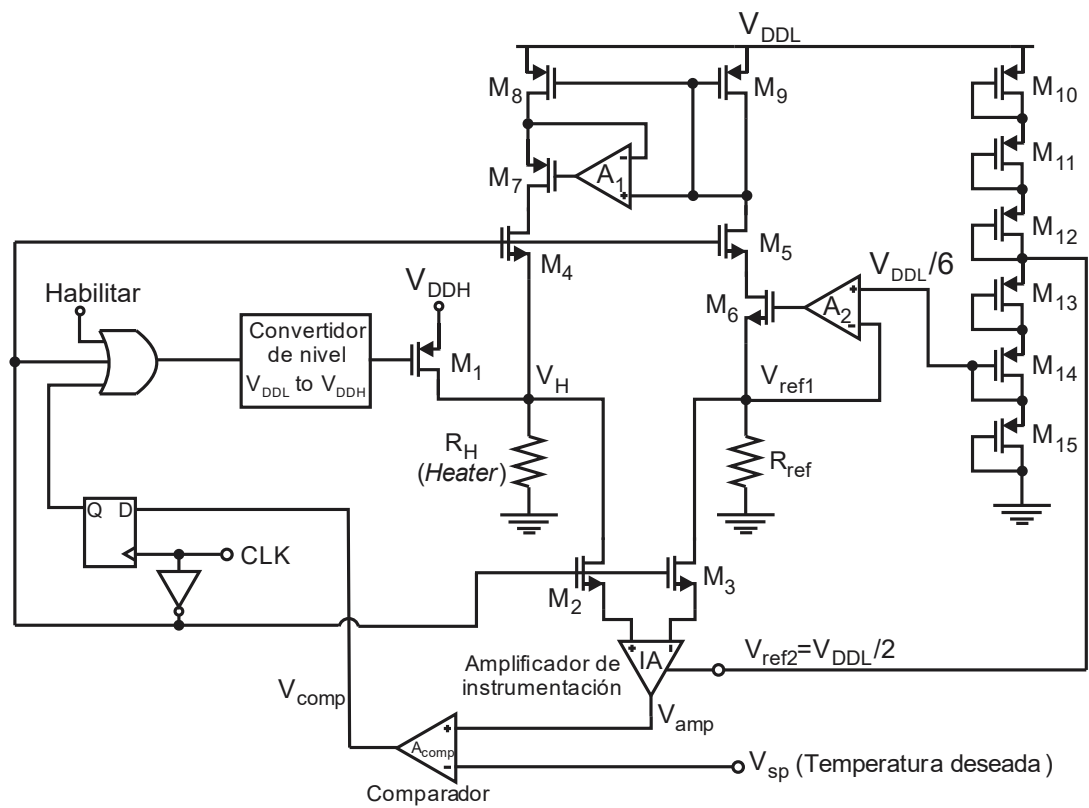

Figura 2. Esquemático del circuito de control de temperatura propuesto

$V_{D D H}$, es el voltaje de alimentación que le proporciona la potencia necesaria para el calentamiento del Heater, el cual puede ser mucho mayor al voltaje $V_{D D L}$. Este trabajo se ha desarrollado en una tecnología CMOS de UMC de $180 \mathrm{~nm}$, por lo que $V_{D D L}$ es de $1.8 \mathrm{~V}$. Esta tecnología cuenta con transistores de alto voltaje que pueden trabajar a $3.3 \mathrm{~V}$, los cuales fueron utilizados en la rama de $V_{D D H}$.

El circuito propuesto desarrolla cuatro acciones principales: medida, comparación, almacenamiento digital y acción de control. Todas ellas sincronizadas por medio de la señal de reloj . La medida de la resistencia del Heater y la comparación con el voltaje de referencia (temperatura deseada) se realizan cuando $C L K=0$. Durante este estado, los interruptores $M_{2}$ y $M_{3}$ se encienden, por lo que conectan al amplificador de instrumentación con el Heater. De igual forma, los interruptores $M_{4}$ y $M_{5}$ conectan al Heater con el espejo de corriente, y finalmente $M_{1}$, al ser de tipo PMOS, se apaga, desconectando al Heater del voltaje $V_{D D H}$. Durante la transición del reloj de 0 a 1, el resultado de la comparación es almacenado en el Flip-Flop tipo D. Finalmente, cuando $C L K=1$, el amplificador de instrumentación y el espejo de corriente son desconectados (ya que los interruptores de $M_{2} a M_{5}$ se apagan), la acción de control es ejecutado a través del transistor $M_{1}$, el cual es encendido o apagado dependiendo del valor almacenado en el FlipFlop ( $0 \rightarrow$ enciende, $1 \rightarrow$ apaga). Lo anterior permite que el Heater se alimente con $V_{D D H}$ si su temperatura es menor al valor deseado, especificado por $V_{s p}$, y se desconecte cuando lo haya superado.

Durante el proceso de medida, el circuito genera una corriente de referencia $l_{r e f}=V_{\text {ref } 1} / R_{r e f}$. El voltaje $V_{\text {ref } 1}$ se obtiene mediante un seguidor de voltaje implementado con el amplificador $\mathrm{A}_{2}$ y el transistor $\mathrm{M}_{6}$, el cual toma el voltaje de referencia $V_{D D L} / 6$ de una cadena de transistores de igual dimensiones conectados en diodo $\left(M_{10}\right.$ a $M_{15}$ ), con una caída de voltaje de $V_{D D H} / 6$ en cada transistor. La corriente de referencia generada $I_{\text {ref }}$ se transfiere 
a la rama del Heater a través del espejo de corriente $\left(M_{7}\right.$ $\left.a M_{9}\right)$, generando el voltaje $V H=V_{r e f 1}\left(R_{H} / R_{r e f}\right)$. Cabe mencionar que en el espejo de corriente se ha incluido el amplificador A1, con el fin de mantener el voltaje $V_{d s}=V_{g s}$ en los transistores M8 y M9 para mejorar la copia de corriente. La diferencia de potencial $\left(V_{H}-V_{\text {ref }}\right)$ es amplificada por el amplificador de instrumentación $I A$, implementado en modo corriente y cuyo esquemático se muestra en la Figura 3, produciendo entonces un voltaje de salida dado por

$$
V_{\text {amp }}=V_{r e f 1}\left[\frac{R_{H}}{R_{r e f}}-1\right] G+V_{r e f 2}
$$

donde $G$ es la ganancia del amplificador y $V_{\text {ref } 2}$ es un voltaje de referencia (obtenido de la cadena de transistores conectados en diodo $M_{10}$ a $M_{15}$ de la Figura 2) que permite fijar el nivel de voltaje de salida $V_{a m p}$ para cuando ambas entradas del amplificador de instrumentación son iguales $\left(V_{H}-V_{\text {ref } 1}\right)$, de esta manera un voltaje de alimentación dual no es necesario. Para que la salida $V_{\text {amp }}$ se encuentre a la mitad del voltaje de alimentación (cuando $R_{H}=R_{H 0}$ ), y a partir de ahí varíe en todo el rango de $V_{D D L}$ para variaciones de $\Delta R_{H}$, el valor de la resistencia $R_{r e q} q u e d a$ determinado por:

$$
R_{r e f}=R_{H 0}+\frac{\Delta R_{H}}{2}
$$

Donde $R_{H 0}$ es el valor de la resistencia del Heater a la temperatura ambiente $T_{A}$ y $\Delta R_{H}$ es la variación de la resistencia del Heater debido a las variaciones de su temperatura de operación. El valor de $R_{H}$ respecto a su temperatura $T_{H}$ puede expresarse de manera simple como

$$
R_{H} R_{H 0}\left[1+\alpha\left(T_{H}-T_{A}\right)\right]
$$

donde $\alpha$ es el coeficiente de temperatura de $R_{H}$ a la temperatura ambiente $T_{A}$ (Lee y Reedy,1999). (Figura 3).

El voltaje de salida del amplificador se compara con $V_{s p}$ el cual representa el valor de temperatura del Heater deseado y por ende la temperatura de operación del sensor, despejando de la Ecuación (3):

$T_{H}=\frac{1}{\alpha}\left[\frac{R_{H}}{R_{H 0}}-1\right]+T_{A}$

La salida del comparador es almacenada en el Flip-Flop tipo $\mathrm{D}$, cuyo valor se usa en la fase de control para activar o desactivar al transistor $M_{1}$ con el fin de mantener la temperatura de operación del sensor alrededor de la temperatura deseada.

La salida de la compuerta $O R$, no puede ser conectada directamente a la compuerta del transistor $M_{1}$, ya que este no se desactivará si la diferencia entre los dos voltajes de alimentación es superior al voltaje de encendido $\left(\left|V_{D D L}-V_{D D H}\right| \geq\left|V_{t p}\right|\right)$. Para evitar que ocurra el caso anterior y aumentar la flexibilidad del esquema propuesto se agrega el acoplador de niveles lógicos mostrado en la Figura 4 (Ishihara et al., 2004). El acoplador ayuda además a manejar la carga capacitiva de la compuerta de $M_{1}$.

\section{Simulaciones y Resultados}

Para mostrar la funcionalidad y desempeño del circuito propuesto se han tomado las características del sensor de gas comercial de monóxido de carbono AS-MLC de la empresa AppliedSensor. Sin embargo, para poder simular el circuito en conjunto con el sensor, es necesario un modelo eléctrico del Heater. En la Figura 5 se muestra la representación del Heater en el dominio eléctrico, donde $R_{H}$ es el valor de su resistencia (dada por la Ecuación 3),

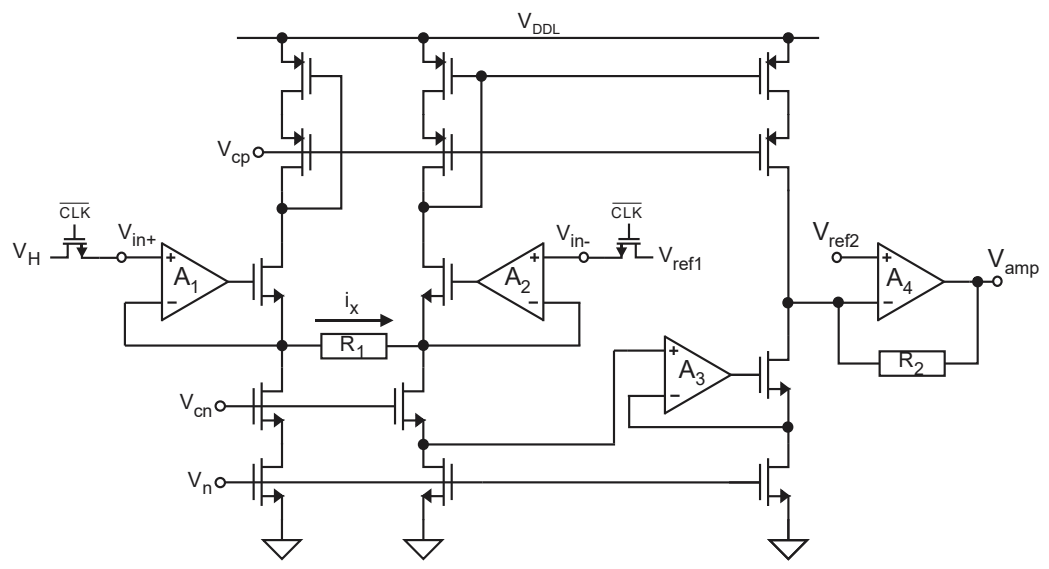

Figura 3. Esquemático del amplificador de instrumentación 

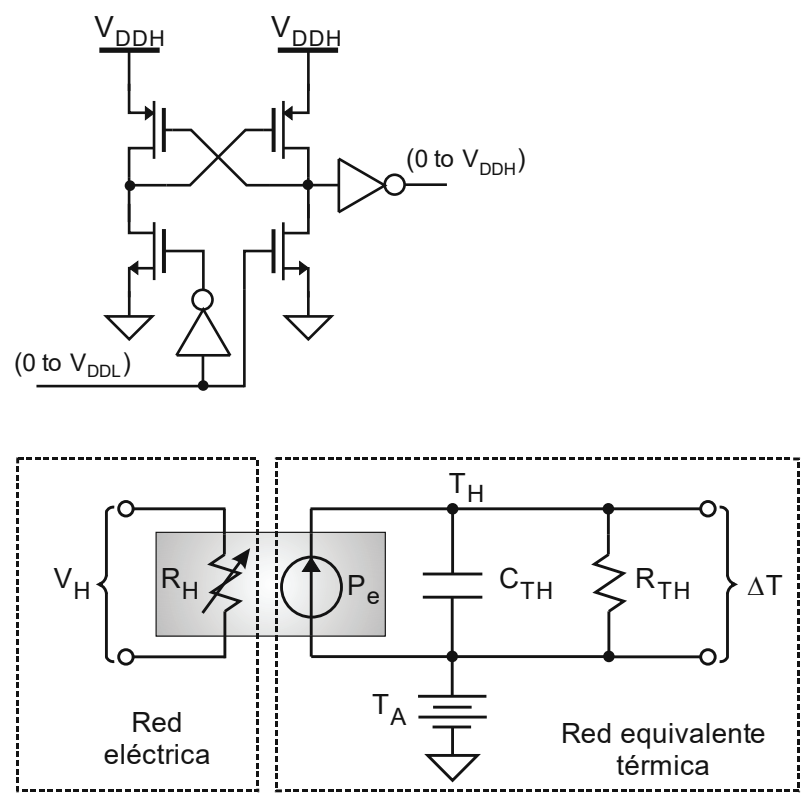

$P_{e}$ es la potencia eléctrica suministrada, $R_{T H}$ y $C_{T H}$ representan la resistencia y capacitancia térmica respectivamente, y la fuente $T_{A}$ la temperatura ambiente. De acuerdo con el modelo eléctrico de la Figura 5, la razón del incremento en la temperatura del calentador queda descrita por la ecuación de equilibrio (Mo et al., 2002):

$\frac{d T_{H}}{d t}=\frac{P_{e}}{C_{T H}}-\frac{T_{H}-T_{A}}{\tau_{T}}$

donde

$P_{e} \quad=$ calor por efecto Joule proporcionada por el voltaje aplicado y

$\tau_{T}=R_{T H} C_{T H}=$ constante de tiempo térmica

La potencia instantánea aplicada al Heater, $P_{e^{\prime}}$ es una función del voltaje aplicado $V_{H} \mathrm{y}$ de la resistencia del Heater $R_{H}$ como se muestra enseguida:

$P_{e}=\frac{\left(V_{H}\right)^{2}}{R_{H}}$

Considerando las ecuaciones (3), (5) y (6) se implementó el modelo eléctrico del Heater mediante fuentes controladas, tal como se muestra en la Figura 6, los cuales fueron descritos en Verilog-AMS para su interconexión y simulación con el circuito propuesto.

El modelo eléctrico fue aplicado al sensor comercial de gas CO AS-MLC de la empresa AppliedSensor, cuyo
Figura 4. Esquemático del convertidor de nivel

Figura 5. Modelo eléctrico del calentador (Heater)

Heater tiene un coeficiente de temperatura de $\alpha=1700 \mathrm{ppm} /{ }^{\circ} \mathrm{K}$ a $T_{A}=20^{\circ} \mathrm{C}$. La resistencia del sensor a temperatura ambiente, $R_{H 0}$ y la constante de tiempo, $\tau_{T}$, fueron medidas experimentalmente. Para determinar la constante de tiempo se aplicó una entrada escalón de $2.3 \mathrm{~V}$ al Heater mientras se monitoreaba su valor resistivo con una SourceMeter Keithley 2400, obteniendo un valor de $22 \mathrm{~ms}$, tiempo en el que alcanza $63.2 \%$ de su valor final. El valor de se midió con el mismo instrumento, pero aplicando un voltaje de solo $10 \mathrm{mV}$ para evitar su autocalentamiento y obtener el valor correcto de su resistencia a temperatura ambiente, obteniendo un valor de $102 \Omega$. Por lo anterior $R_{T H}$ y $C_{T H}$ del modelo se establecieron en $6.3 \mathrm{k} \Omega$ y $3.47 \mu \mathrm{F}$, respectivamente.

El circuito propuesto fue simulado aplicando diferentes señales de entrada, $V_{s p}$, con las formas más representativas de las técnicas de modulación de la temperatura de operación de sensores de gas MOX (Lee y Reedy, 1999). La señal de reloj se estableció a una frecuencia de 10kHz. En la Figura 7 se observa la respuesta del circuito propuesto bajo una entrada escalón en la señal de referencia, $V_{s p}$, de tal manera que el Heater cambia de la temperatura ambiente a una temperatura de operación particular. En la Figura 7a se muestra la resistencia del Heater desde su valor a temperatura ambiente $R_{H}=102 \Omega$, hasta el valor final $R H=135 \Omega$; lo que corresponde a un cambio en la temperatura de operación del sensor de $20{ }^{\circ} \mathrm{C}$ a aproximadamente $210{ }^{\circ} \mathrm{C}$ (Figura $7 b$ ). En la misma figura se muestra también un acercamiento de la parte en donde la resistencia se ha establecido alrededor del valor deseado. El rizo obtenido es inferior a $0.2 \Omega$, lo que corresponde a un error 


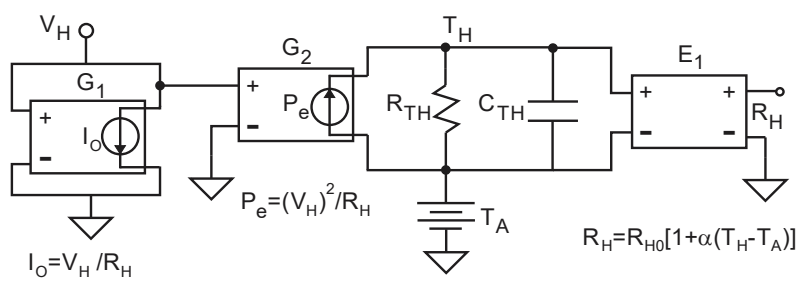

(a) Resistencia del Calentador
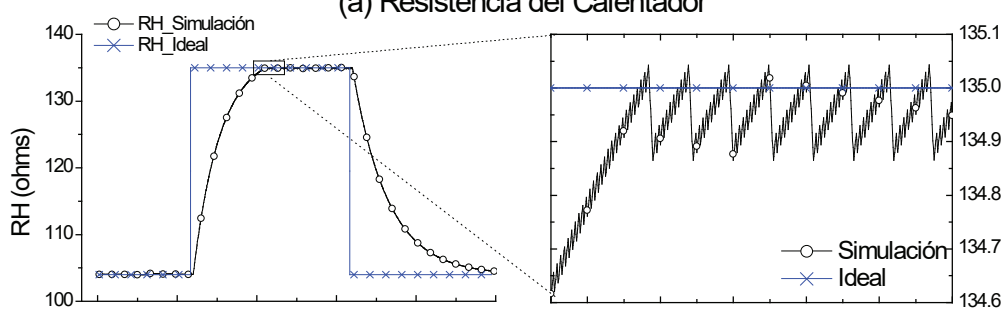

(b) Temperatura del Calentador

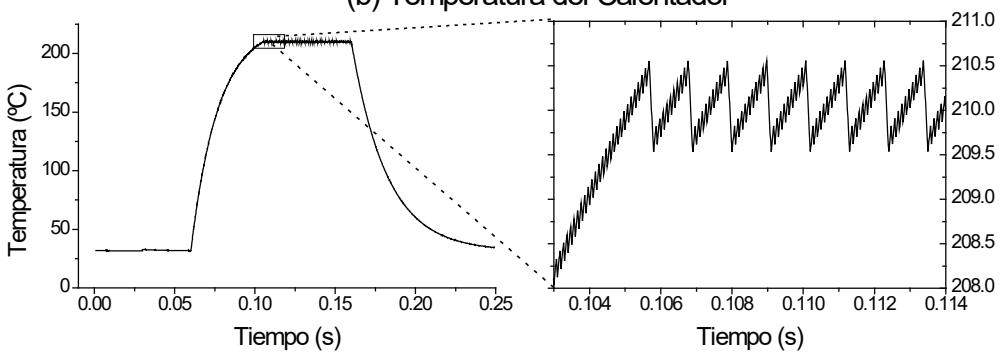

Figura 6. Implementación del modelo eléctrico del calentador con fuentes controladas aproximado de $1^{\circ} \mathrm{C}$ en la temperatura de operación del sensor.

Un arreglo virtual de sensores de gas puede ser generado usando un solo sensor; esto es posible porque los sensores de gas MOX son sensibles a diferentes gases utilizando distintas temperaturas de operación. Por lo anterior, el circuito fue simulado para controlar la temperatura de operación a diferentes niveles. En la Figura 8 se muestra la salida del circuito de control bajo una entrada que simula los diferentes niveles de temperatura de operación. Se puede observar que la temperatura se establece en los valores deseados después de cierto tiempo debido a la constante de tiempo térmica del propio sensor, en este caso de $22 \mathrm{~ms}$.

Finalmente, se realizaron simulaciones con una señal de entrada sinusoidal de $2 \mathrm{~Hz}$ para verificar el uso del circuito en las técnicas de modulación de temperatura siguiendo una forma de onda periódica. En la Figura 9 se muestra el resultado de la simulación, se puede observar, que una vez que el calentador alcanza el valor deseado, este sigue la forma de onda. Los mejores resultados para discriminar gases o incrementar la selectividad de los sensores MOX se han encontrado con frecuencias de modulación de temperatura por debajo de $1 \mathrm{~Hz}$ (Sun et al., 2004), por lo que el circuito de control de temperatura propuesto puede ser usado para esas aplicaciones.

Cabe mencionar que en todas y cada una de las simulaciones, el error en el control de la resistencia se mantuvo por debajo de $0.2 \Omega$, lo que significa que el error en la temperatura de operación fue de aproximadamente $1{ }^{\circ} \mathrm{C}$. Para el sensor usado, la potencia promedio en la fase de medida fue de $1.7 \mathrm{~mW}$ mientras que en el periodo de calentamiento fue de $43.6 \mathrm{~mW}$.

En la Tabla 1 se proporciona un concentrado de las principales características del circuito de control de temperatura y una comparación con los trabajos más representativos de las técnicas de control de temperatura para sensores de gas de tipo MOX. Con el esquema propuesto se consigue la simplicidad y eficiencia de una técnica de control on/off y la flexibilidad en cuanto a voltajes de alimentación de un sistema que usa técnicas de control clásico.

Para demostrar la funcionalidad de la arquitectura del circuito propuesto, el circuito de control fue fabricado con la tecnología UMC 180nm CMOS. El layout y la fotografía del sistema se muestran en la Figura 10. El área del circuito integrado fue de $0.06 \mathrm{~mm}^{2}$.

El circuito fabricado fue probado con señales escalón introducidas en $V_{s p}$ (ver Figura 2) y como señal de 

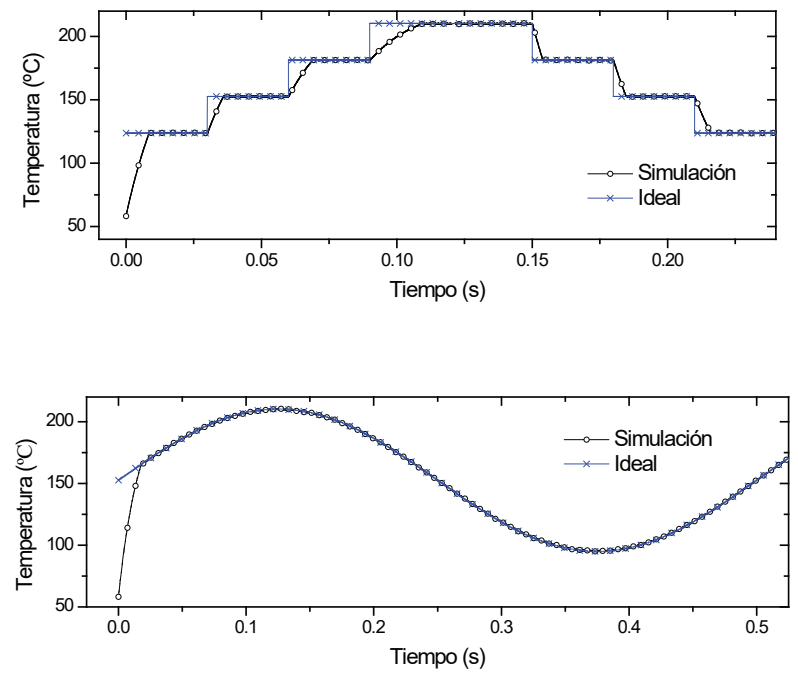

Figura 8. Respuesta del circuito de control bajo diferentes niveles de referencia de entrada

Figura 9. Respuesta del circuito de control a una entrada con forma de onda periódica

Tabla 1. Comparación de los circuitos de control de temperatura

\begin{tabular}{cccc}
\hline Parámetro & Circuito propuesto & $\begin{array}{c}{[14]} \\
\text { On/off }\end{array}$ & $\begin{array}{c}\text { [15] } \\
\text { Control PID }\end{array}$ \\
\hline Tecnología & $180 \mathrm{~nm}$ & - & $800 \mathrm{~nm}$ \\
VDD & $1.8 \mathrm{~V} \mathrm{y} 3.3 \mathrm{~V}$ & $5 \mathrm{~V}$ & $5 \mathrm{~V}$ y $15 \mathrm{~V}$ \\
Resolución (simulado) & $\pm 1^{\circ} \mathrm{C}$ & $\pm 1^{\circ} \mathrm{C}$ & $1^{\circ} \mathrm{C}$ \\
Resistencia del Heater & $102-150 \Omega$ & $50-100 \Omega$ & $50-100 \Omega$ \\
Consumo de potencia & $\sim 1.7 \mathrm{~mW}$ & $\sim 2.18 \mathrm{~mW}$ & $-800 \Omega$ \\
Manejo de heaters con diferente potencia & $\mathrm{Si}$ & $\mathrm{No}$ & $\mathrm{Si}$ \\
Complejidad & Baja & Baja & Alta \\
\hline
\end{tabular}

salida fue monitoreado $V_{a m p}$, cuyo valor representa la temperatura de operación deseada de acuerdo con la relación definida en la ecuación (1). La Figura 11 muestra la respuesta del sistema cuando $V_{s p}$ cambia de $0.5 \mathrm{~V}$ a $0.95 \mathrm{~V}$ y la Figura 12 cuando la entrada cambia de 0.95 a $0.5 \mathrm{~V}$ en ambos casos el tiempo de establecimiento fue de aproximadamente $45 \mathrm{~ms}$. De acuerdo con las relaciones definidas en las ecuaciones (1) y (4) el cambio producido corresponde a una variación de temperatura de $61^{\circ} \mathrm{C}$ a $164^{\circ} \mathrm{C}$.

La funcionalidad del circuito de control se muestra en la Figura 13. El rango de tiempo mostrado corresponde al estado estacionario, cuando la resistencia del Heater ha llegado al valor deseado, después de la parte transitoria mostrada en las Figuras 11 y 12. La frecuencia del reloj se estableció a $3 \mathrm{kHz}$ con un ancho de pulso de $60 \%$. Tal como se puede observar, cuando el voltaje de salida del amplificador de instrumentación $V_{a m p}$ es menor que el voltaje de referencia $V_{s p}$, el circuito de control aplica al Heater, el voltaje máximo $V_{D D H}($ en $V H$ ) para calentar el sensor. Una vez que la temperatura del sensor sobrepasa el valor deseado $\left(V_{a m o}>V_{s p}\right)$ el circuito de control desconecta al Heater de $V_{D D H}$ manteniendo de esta manera la temperatura del sensor alrededor del valor deseado. El sistema estableció la temperatura de operación del sensor en el valor deseado con un error de $\pm 5.4^{\circ} \mathrm{C}$. Este error es más alto que el obtenido en las simulaciones debido a que la frecuencia de reloj usado en la parte experimental fue de solo $3 \mathrm{kHz}$, mientras que en las simulaciones se usó un reloj de $10 \mathrm{kHz}$. Se empleó una frecuencia de reloj más bajo con el propósito de poder observar con mayor claridad el funcionamiento del sistema (mostrado en la Figura 13). Cabe destacar entonces que el error se reduce al aumentar la frecuencia del reloj, así como reduciendo el nivel de ruido de los voltajes de referencia. 
a) Layout $\quad$ b) Fotografía del circuito integrado
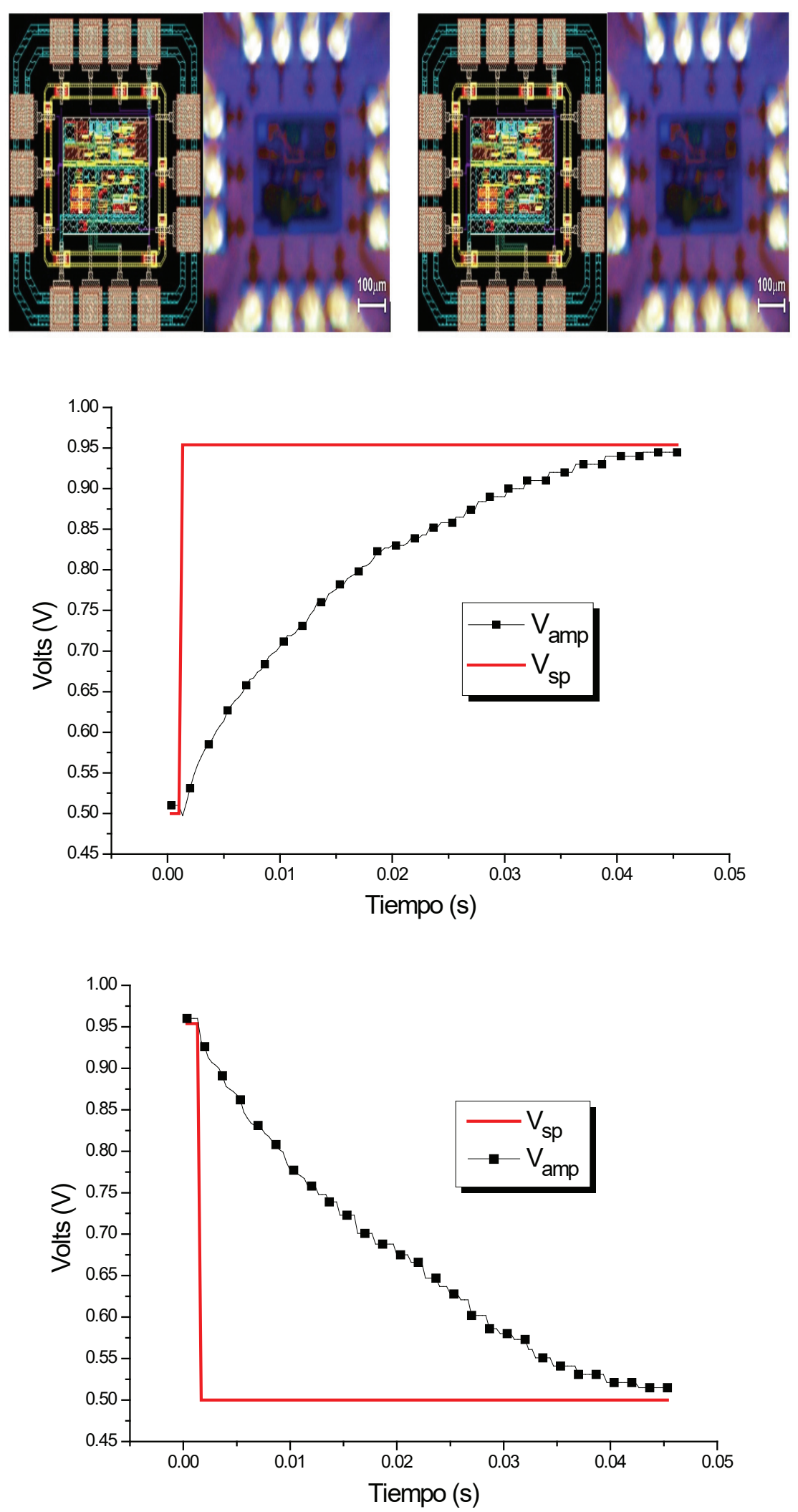

Figura 10. Implementación del circuito integrado

Figura 11. Respuesta experimental del circuito de control a un cambio del voltaje de entrada de 0.5 a $0.95 \mathrm{~V}$ (correspondiente a un cambio de $61^{\circ} \mathrm{a}$ $\left.164^{\circ} \mathrm{C}\right)$

Figura 12. Respuesta experimental del circuito de control a un cambio del voltaje de entrada de 0.5 a $0.95 \mathrm{~V}$ (correspondiente a un cambio de 164 a $\left.61^{\circ} \mathrm{C}\right)$ 


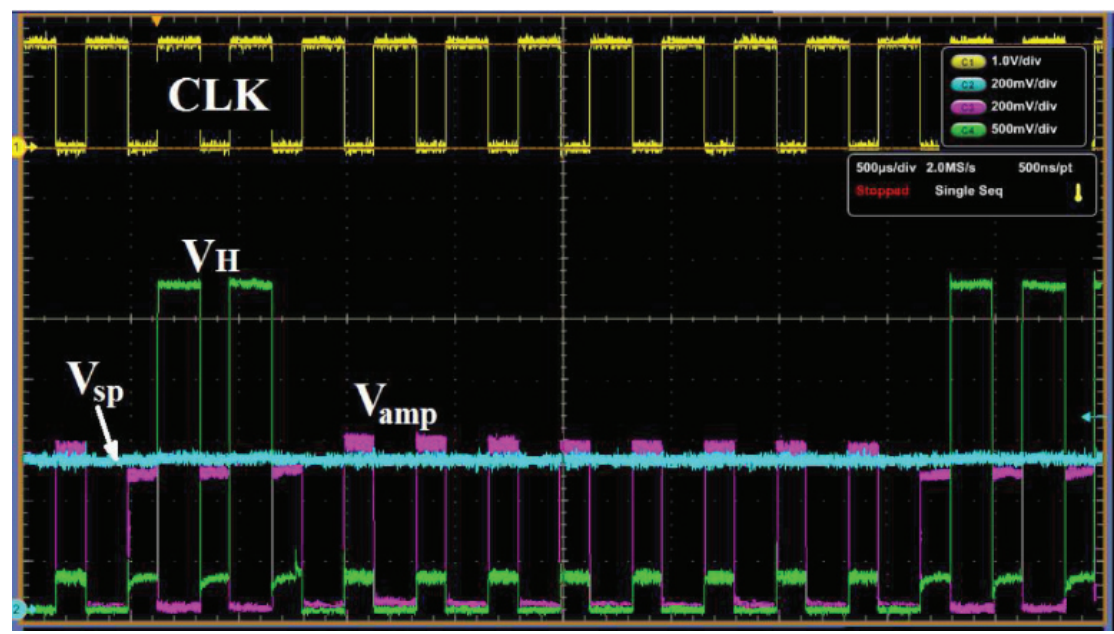

Figura 13. Respuesta experimental que muestra el modo de operación del circuito de control de temperatura: voltaje de set-point $\left(V_{s p}\right)$, Voltaje de salida del amplificador de instrumentación $\left(V_{\text {amp }}\right)$, Voltaje aplicado al Heater $\left(V_{H}\right)$ y señal de reloj $(C L K)$

\section{Conclusiones}

En este trabajo se ha propuesto un circuito de control on/off que utiliza la resistencia del propio calentador como sensor de temperatura. En contraste con otros esquemas propuestos en la literatura, el circuito propuesto adopta la simplicidad de un esquema de control on/off y adiciona la capacidad de manejar sensores de gas MOX con diferentes requerimientos de potencia al tener la flexibilidad para manejar dos niveles de voltaje; uno para los circuitos de acondicionamiento de señal, y otro exclusivo para la alimentación del Heater. Lo anterior abre la posibilidad de poder migrar a otras tecnologías de fabricación, con el único requerimiento de que la tecnología cuente con transistores de alto voltaje para manejar la potencia requerida por el Heater.

Por otra parte, se demostró que el circuito de control permite aplicar las técnicas de modulación de la temperatura de operación de los sensores MOX que han sido reportados en la literatura para la mejora de su selectividad: modulación por pulsos, generación de matrices virtuales de sensores mediante un solo sensor y modulación de la temperatura siguiendo una determinada forma de onda periódica, entre otras. El control en la resistencia del calentador permitió obtener un error inferior a $0.2 \Omega$, que corresponde a un error en la temperatura de operación de aproximadamente $1{ }^{\circ} \mathrm{C}$.

\section{Agradecimientos}

Los autores agradecen al CONACyT por el apoyo recibido a través de la beca doctorado 212441 y el proyecto de investigación CB-SEP-2008-01-99901188.

\section{RefERENCIAS}

Bermak, A., Belhouari, S., Shi, M., Martínez, D. (2005). Pattern recognition techniques for odor discrimination in gas sensor array. En Encyclopedia of Sensors, E.C.D. Craig A. Grimes y M.V. Pishko, Eds. American Scientific Publishers.

Bharat, S., Ashutosh, S., Jung-Sik, K. (2018). Recent advances on H2 sensor technologies based on MOX and FET devices: A review. Sensors and Actuators B, 262, pp. 758-770. Recuperado de https://www.x-mol.com/paperRedirect/558360

Bota, S., Dieguez, A., Merino, J., Casanova, R., Samitier, J., Cané, C. (2004). A monolithic interface circuit for gas sensor arrays: Control and measurement. Analog Integrated Circuits and Signal Processing, 40(2), 175-184.

Burgues, J. y Marco, S. (2018). Low power operation of temperature-modulated metal oxide semiconductor gas sensors. Sensors, 18, 339. https://doi.org/10.3390/s18020339

Casanova, R., Merino, J.L., Dieguez, Á., Bota, S.A., Samitier, J. (2004). A mixed-mode temperatura control circuit for gas sensors. En ISCAS (4)'04, pp. 896-909.

Dongmin, G., Zhang, D., Li, N., Zhang, L., Yang, J. (2010). A novel breath analysis system based on electronic olfaction. IEEE Transactions on Biomedical Engineering, 57(11). https://doi. org/10.1109/TBME.2010.2055864

Falconi, C. Systematic design of micro-resistors for temperature control by quasi-simultaneous heating and temperature sensing. Sensors and Actuators B, 179, pp. 336-346, 2013.

Falconi, C. y Fratini, M. (2008). CMOS microsystems temperature control. Sensors and Actuators B: Chemical, 129, 59-66. http:// dx.doi.org/10.1016/j.snb.2007.07.071

Gardner, J.W., Prasanta, K.G., Florin, U., James, A. (2010). Covington. CMOS Interfacing for Integrated Gas Sensors: A Review. IEEE Sensors Journal, 10(12). 
Ishihara, F., Sheikh, F., Nikolic, B. (2004). Level conversion for dualsupply systems. Very Large Scale Integration (VLSI) Systems. IEEE Transactions On, 12(2), 185-195. https://doi. org/10.1109/TVLSI.2003.821548

Jendritzky G. (1993). The atmospheric environment-an introduction. Experientia 49. Basel/Switzerland: Birkhaiuser Verlag.

Kang, J. et al. (2017). Temperature control of heater using Pt thin film temperatura sensor embedded in micro gas sensor, Micro and Nano Systems Letters, Vol. 5, pp. 26. https://doi. org/10.1186/s40486-017-0060-z

Korotcenkoy, G. (2007). Metal oxides for solid-state gas sensors: What determines our choice? Materials Science and Engineering: B, 139(1), 1-23. https://doi.org/10.1016/j.mseb.2007.01.044

Lee, A.P. y Reedy, B.J. (1999). Temperature modulation in semiconductor gas sensing. Sensors and Actuators B, 60, 35-42. https://doi.org/10.1016/S0925-4005(99)00241-5
Mo, Y., Okawa, Y., Inoue K., Natukawa K. (2012). Low-voltage and low-power optimization of micro-heater and its on-chip drive circuitry for gas sensor array. Sensors and Actuators A: Physical, 100, 94-101. http://dx.doi.org/10.1016/S09244247(02)00145-0

Ponzoni, A. et al. (2017). Metal oxide gas sensors, a survey of selectivity issues addressed at the SENSOR Lab, Brescia (Italy). Sensors (Basel, Switzerland), 17(4), 714. https://dx.doi. org/10.3390\%2Fs17040714

Seiyama, T., Kato, A., Fujiishi, K., Nagatani, M. (1962). A new detector for gaseous components using semiconductive thin films. Analytical Chemistry, 34(11), 1502-1503.

Sun, Y., Huang, X., Meng, F.,Liu, J. (2004). Study of influencing factors of dynamic measurements based on $\mathrm{SnO} 2$ gas sensor. Sensors, 4(6), 95-104. https://doi.org/10.3390/s40670095 